\title{
Short-Term Hydro Scheduling considering Risk Aversion in a MINLP Approach
}

\author{
J.P.S. Catalão ${ }^{1}$, H.M.I. Pousinho ${ }^{1}$ and V.M.F. Mendes ${ }^{2}$ \\ ${ }^{1}$ Department of Electromechanical Engineering \\ University of Beira Interior \\ R. Fonte do Lameiro, 6200-001 Covilhã (Portugal) \\ Phone: +351 275 329914, Fax: +351 275 329972, e-mail: catalao@ubi.pt, hmi-21@hotmail.com \\ ${ }^{2}$ Department of Electrical Engineering and Automation \\ Instituto Superior de Engenharia de Lisboa \\ R. Conselheiro Emídio Navarro, 1950-062 Lisbon (Portugal) \\ Phone: +351 218317 000, Fax: +351218317 001, e-mail: vfmendes@isel.pt
}

\begin{abstract}
This paper presents an optimization model to help a hydro generating company to schedule its hydroelectric units in the short-term under a competitive environment. The hydro generating company considered has the ultimate goal of maximizing profits, taking also into account some risk aversion criterion. The problem is formulated as a mixed-integer nonlinear programming problem. An application to a real case study is presented. Conclusions are duly drawn.
\end{abstract}

\section{Key words}

Hydro scheduling, competitive environment, risk averse, mixed-integer nonlinear programming.

\section{Introduction}

In this paper, the short-term hydro scheduling (STHS) problem of a head-dependent hydro chain is considered. Hydro plants with only a small storage capacity available are known as run-of-the-river. Due to the reservoirs small storage capacity, the operating efficiency becomes sensitive to the head-head change effect. The cascaded hydraulic configuration, coupled with the nonlinear head change effect, augments the problem dimension and the complexity.

The main goal in the STHS problem is to maximize the value of total hydroelectric power generation throughout the time horizon, satisfying all physical and operational constraints, and consequently to maximize the profit of the hydro generating company from selling energy into the electric market [1]. Also, as a new contribution to earlier studies, a risk aversion criterion is taken into account.

Indeed, any producer should self-schedule its units to maximize the expected profit assuming a given level of risk. This self-schedule is then used by the producer to derive an appropriate bidding strategy to the pool [2].
Mixed-integer linear programming (MILP) is becoming often used for STHS [3, 4], where integer variables allow modeling of start-up costs, which are mainly caused by the increased maintenance of windings and mechanical equipment, and by malfunctions of the control equipment.

In this paper, a mixed-integer nonlinear programming (MINLP) approach is proposed to solve the STHS problem. The proposed approach considers not only head-dependency but also discontinuous operating regions. We report our experience with the proposed approach on a real case study.

\section{Proposed Approach}

\section{A. Objective Function}

In this paper, the objective function takes into account all the price scenarios at once, weighed by their occurrence probability:

$$
\max \sum_{n=1}^{N} \rho_{n} B_{n}+\alpha\left(\zeta-\frac{1}{1-\delta} \sum_{n=1}^{N} \rho_{n} \eta_{n}\right)
$$

where $\rho_{n}$ is the probability associated to the scenario $n$, $\alpha$ is the weighting positive factor to achieve appropriate trade-off profit versus risk, $\zeta$ is the Value at Risk at a confidence level of $\delta, \eta$ is an auxiliary variable used to compute the conditional value at risk $(\mathrm{CVaR})$ and $B_{n}$ is the benefit for each price scenario calculated as:

$$
B_{n}=\lambda_{k n} \sum_{i=1}^{I} p_{i k}
$$

where $\lambda_{k n}$ is the price for scenario $n$ at the hour $k ; p_{i k}$ is the hourly electricity production of the hydro unit $i$. 


\section{B. Conditional Value at Risk}

$\mathrm{CVaR}$ is an alternative risk assessment tool that does quantify the losses associated with the tail of the profit distribution. The $\mathrm{CVaR}$ is the expected profit not exceeding a measure called Value at Risk:

$$
C V a R=E(B \mid B<\zeta)
$$

Value at Risk, $\zeta$, is a measure defined as the maximum profit value such that the probability of the profit being lower than or equal to this value than or equal to $1-\delta$ :

$$
\operatorname{VaR}=\max (x \mid \mathrm{p}\{B \leq x\} \leq 1-\delta)
$$

The literature refers that $\delta$ assumes values usually between 0.9 and 0.99 [5]. In this paper, $\delta$ is equal to 0.95. Mathematically, CVaR can be defined as:

$$
\max \zeta-\frac{1}{1-\delta} \sum_{n}^{N} \rho_{n} \eta_{n}
$$

subject to:

$$
\begin{gathered}
-B_{n}+\zeta-\eta_{n} \leq 0 \\
\eta_{n} \geq 0
\end{gathered}
$$

In constraint (6) the $\eta_{n}$ is a variable whose value is equal to zero if the scenario $n$ has a profit greater than $\zeta$. For the rest of scenarios, $\eta_{n}$ is equal to the difference of $\zeta$ and the corresponding profit.

\section{Hydro Constraints}

1) Water Balance: The water balance equation for each reservoir is formulated as:

$$
v_{i k}=v_{i, k-1}+a_{i k}+\sum_{m \in M_{i}}\left(q_{m k}+s_{m k}\right)-q_{i k}-s_{i k}
$$

assuming that the time required for water to travel from a reservoir to a reservoir directly downstream is less than the one hour period.

2) Head: The head is considered a function of the water levels in the upstream and downstream reservoirs:

$$
h_{i k}=l_{f(i) k}\left(v_{f(i) k}\right)-l_{t(i) k}\left(v_{t(i) k}\right)
$$

3) Power Generation: Power generation is considered a function of water discharge and hydro power efficiency:

$$
p_{i k}=q_{i k} \eta_{i k}\left(h_{i k}\right)
$$

4) Water Storage: Water storage has lower and upper bounds:

$$
\underline{v}_{i} \leq v_{i k} \leq \bar{v}_{i}
$$

5) Water Discharge: Water discharge has lower and upper bounds

$$
u_{i k} \underline{q}_{i} \leq q_{i k} \leq u_{i k} \bar{q}_{i}
$$

6) Water Spillage: We consider a null lower bound for water spillage

$$
s_{i k} \geq 0
$$

Water spillage can occur when without it the water storage exceeds its upper bound, so spilling is necessary due to safety considerations. The initial water storages and inflows to reservoirs are assumed known.

\section{Mixed-Integer Nonlinear Programming}

Power generation is considered a nonlinear function of water discharge and water storage, given by:

$$
p_{i k}=\alpha_{i} \beta_{f(i)} q_{i k} v_{f(i) k}-\alpha_{i} \beta_{t(i)} q_{i k} v_{t(i) k}+\chi_{i} q_{i k}
$$

with

$$
\chi_{i}=\alpha_{i}\left(l_{f(i)}^{0}-l_{t(i)}^{0}\right)+\eta_{i}^{0}
$$

A major advantage of our approach is to consider the head change effect in a single function (14) of water discharge and water storage that can be used in a straightforward way, instead of deriving several curves for different heads.

\section{Case Study}

The MINLP approach has been applied on a Portuguese cascaded hydro system. The realistic hydro chain has three cascaded reservoirs and is shown in Figure 1.
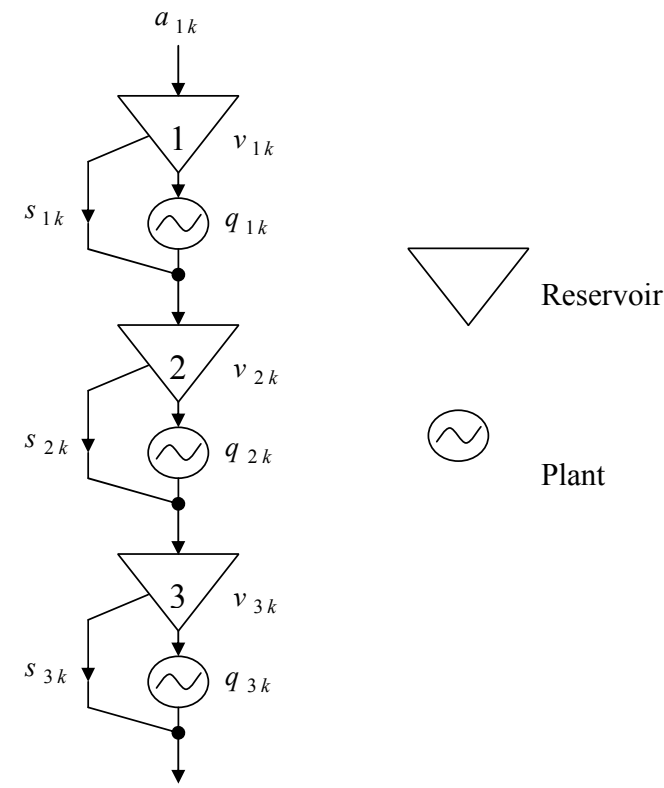

Fig. 1. Cascaded hydro system. 
Our model was implemented on a 600-MHz-based processor with $256 \mathrm{MB}$ of RAM using the optimization solver package Xpress-MP under MATLAB. The scheduling time horizon chosen is one day divided into 24 hourly periods.

The prices scenarios over the time horizon are shown in Figure 2 (where $\$$ is a symbolic economic quantity). The number of prices scenarios generated in the optimization problem is $\mathrm{N}=5$. This number has been selected arbitrarily, and the probability of each generated scenario will be $1 / \mathrm{N}$.

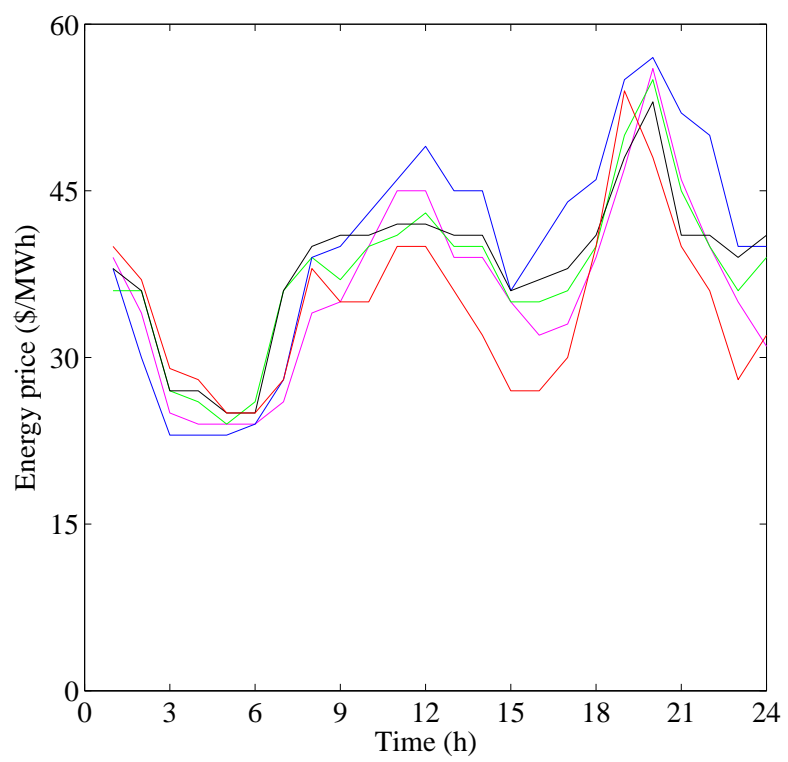

Fig. 2. Prices scenarios considered in the case study.

In restructured markets, price forecasting is extremely important for all market players. An accurate forecast of energy prices has become a very important tool for a generation company to develop an appropriate bidding strategy in the market and to optimally schedule its hydro resources. Several techniques have been tried out for energy prices forecasting [6], mainly based on time series models [7, 8], or on artificial intelligence techniques $[9,10]$. In this case study, the prices scenarios are obtained using the neural networks approach in [10]. Afterwards, the price scenarios are used in the scheduling problem.

The expected profit and the profit standard deviation obtained for four values of $\alpha$ are presented in Figure 3. An analysis of Figure 3, known as efficient frontier, reveals that for a risk-neutral producer $(\alpha=0)$, the expected profit is $\$ 406,569$ with a standard deviation of $\$ 28,038$. On the other hand, a risk averse producer $(\alpha=1)$ expects to achieve a profit of $\$ 404,455$ with a lower standard deviation of $\$ 23,480$.

Table I shows the scheduling results for two levels of risk in the third hydro plant of this case study. The values of $\alpha$ considered are maximum risk $(\alpha=0)$ and a lower level of risk $(\alpha=1)$.

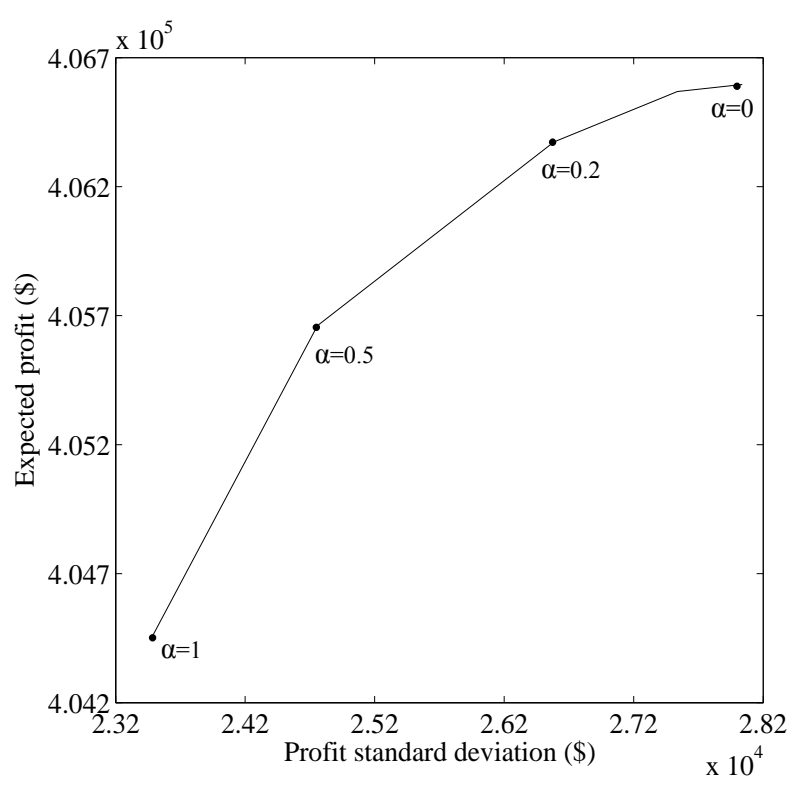

Fig. 3. Expected profit versus profit standard deviation.

Table I. - Scheduling Results for Two Levels of Risk Plant 3

\begin{tabular}{|c|c|c|c|c|c|}
\hline \multirow{2}{*}{ Hour } & $\begin{array}{c}\text { Power generation } \\
\text { per water discharge } \\
{\left[\mathrm{MWh} / \mathrm{hm}^{3}\right]}\end{array}$ & \multirow{2}{*}{ Hour } & \multicolumn{2}{|c|}{$\begin{array}{c}\text { Power generation } \\
\text { per water discharge } \\
{\left[\mathrm{MWh} / \mathrm{hm}^{3}\right]}\end{array}$} \\
\cline { 2 - 3 } \cline { 5 - 6 } & $\alpha=0$ & $\alpha=1$ & & $\alpha=0$ & $\alpha=1$ \\
\hline 1 & 191.74 & 191.74 & 13 & 190.58 & 190.38 \\
\hline 2 & 0 & 191.55 & 14 & 190.38 & 190.25 \\
\hline 3 & 0 & 0 & 15 & 0 & 0 \\
\hline 4 & 0 & 0 & 16 & 0 & 0 \\
\hline 5 & 0 & 0 & 17 & 0 & 0 \\
\hline 6 & 0 & 0 & 18 & 192.03 & 191.14 \\
\hline 7 & 0 & 0 & 19 & 191.83 & 190.95 \\
\hline 8 & 191.55 & 191.35 & 20 & 191.64 & 190.75 \\
\hline 9 & 191.35 & 191.16 & 21 & 191.44 & 190.56 \\
\hline 10 & 191.16 & 190.96 & 22 & 191.25 & 190.36 \\
\hline 11 & 190.97 & 190.77 & 23 & 0 & 0 \\
\hline 12 & 190.77 & 190.57 & 24 & 191.94 & 0 \\
\hline
\end{tabular}

The results obtained in Table I show that different risk levels provide a different scheduling, i.e., in time periods in which hydro plant is online $(\alpha=1)$ the production is considerably lower compared to the case of maximum risk $(\alpha=0)$.

\section{Conclusion}

In this paper, the risk measure $\mathrm{CVaR}$ is included in the optimal hydro scheduling problem. The objective function is the maximization of the expected total profit plus a risk measure of the profit. The problem is formulated as a mixed-integer nonlinear problem that is solved using the optimization solver package Xpress-MP under MATLAB. The numerical results provided by this approach include the efficient frontier curve, giving the expected profit vs. profit standard deviation. The efficient frontier curves are important to make informed decisions on the pool. 


\section{References}

[1] J.P.S. Catalão et al., "Scheduling of head-sensitive cascaded hydro systems: a nonlinear approach", IEEE Trans. Power Syst., Vol. 24, pp 337-346, 2009.

[2] A.J. Conejo et al., "Risk-constrained self scheduling of a thermal power producer", IEEE Trans. on Power Syst., Vol. 19, pp 1569-1574, 2004.

[3] J. García-González et al., "Risk-averse profit-based optimal scheduling of a hydro-chain in the day-ahead electricity market”, Eur. J. Oper. Res., Vol. 181, pp 13541369,2007

[4] A. Borghetti et al., "An MILP approach for short-term hydro scheduling and unit commitment with headdependent reservoir", IEEE Trans. Power Syst., Vol. 23, pp 1115-1124, 2008.

[5] A.J. Conejo et al., "Optimal involvement in futures markets of a power producer", IEEE Trans. Power Syst., Vol. 23, pp 701-711, 2008.
[6] S.K. Aggarwal, L.M. Saini, and A. Kumar, "Electricity price forecasting in deregulated markets: a review and evaluation", Electr. Power Energy Syst., Vol. 31, pp 1322, 2009.

[7] A.J. Conejo et al., "Day-ahead electricity price forecasting using the wavelet transform and ARIMA models", IEEE Trans. Power Syst., Vol. 20, pp 1035-1042, 2005.

[8] J. Contreras et al., "ARIMA models to predict next-day electricity prices", IEEE Trans. Power Syst., Vol. 18, pp 1014-1020, 2003.

[9] N. Amjady and F. Keynia, "Day ahead price forecasting of electricity markets by a mixed data model and hybrid forecast method", Electr. Power Energy Syst., Vol. 30, pp 533-546, 2008.

[10] J.P.S. Catalão et al., "Short-term electricity prices forecasting in a competitive market: a neural network approach", Electr. Power Syst. Res., Vol. 77, pp 1297 12304, 2007. 